\title{
Hypothesis: Mycotoxins Causing Amyotrophic Lateral Sclerosis -Immune Suppression \& Immunotherapy
}

\author{
William Reid ${ }^{1}$ \\ ${ }^{1}$ Affiliation not available
}

March 16, 2021

Wednesday, March 3, 2021

William Reid

Amyotrophic Lateral Sclerosis(ALS) remains a terminal disease without an established etiology for the majority of patients. The dominant theory of ALS before 1970's was the presence of poisons. One of the primary means of treating patients with a toxic exposure has been plasma exchange, but plasma exchange of ALS patients failed to alter the clinical course. The failure of plasma exchange assumes the patient is no longer exposed to the poison. If the toxic exposure continues after plasma exchange, then plasma exchange alone would fail. The world food supply is contaminated by opportunistic fungi that release mycotoxins. There is mounting evidence of the profound neurologic and immunologic toxicity of the most common mycotoxins. Every ALS patient examined had evidence of a toxic exposure. Treatment with antifungals specific to the most common opportunistic fungi led to improvement in laboratory evidence of a poisoning. The pathology of the common mycotoxins mimics the pathology reported in ALS patients. Along with the profound neurotoxicity of mycotoxins, there is profound immune toxicity. If mycotoxins are involved causing ALS, then the immunotoxicity of mycotoxins could be responsible for the recalcitrance of therapy reported in ALS. New forms of treatment for immune deficits, immune tolerance and immune evasion could remarkably improve the treatment of ALS patients.

\section{Introduction :}

The etiology of ALS has remained elusive. The dominant theory before the 1970's was the presence of neurotoxins ${ }^{1,2}$. Serum from patients with ALS were toxic to motor neuron cells in culture. Based on this theory multiple institutions treated ALS patients with plasma exchange without apparent improvement ${ }^{3}$. The conclusion that plasma exchange was ineffective made a critical assumption that there is a finite body burden of neurotoxin and no further exposures. If, instead exposure to neurotoxins persist after plasma exchange then plasma exchange would be inadequate. The source of toxins whether environmental or endogenous would need to be removed. If neurotoxins were generated by an infectious agent colonizing or infecting patients, then treatment would require control of the infection along with plasma exchange.

In every ALS patient studied, there was laboratory evidence of a poisoning ${ }^{4}$. Patients developed secondary porphyria in a pattern typical of a poisoning. They developed anion-gap positive metabolic acidosis. They all had elevated Kreb cycle intermediates consistent with mitochondrial damage typically seen with toxic exposures. Treatment with anti-fungal agents specific to Fusarium species corrected many of the laboratory findings including the elevated protoporphyrins, the anion-gap positive metabolic acidosis and the elevated Kreb cycle intermediates. This is consistent with the presence of mycotoxin generated by a fungus growing in the patient.

The literature on mycotoxins is extensive $e^{5-7}$. Mycotoxins are ubiquitous in the human environment and prominent in the world food supply. Globalization has accentuated the problem. Efforts to prevent or 
remove fungi and their mycotoxins from food and the environment have been difficult to impossible. Humans are routinely colonized by fungi that populate the skin, gastrointestinal tract, lungs and upper airway ${ }^{8,9}$. The same fungal species contaminating food are the species colonizing the human airways. Surveys of grain supplies found hundreds of fungal species producing over 700 mycotoxins $^{5}$. Four fungal genera were routinely reported Aspergillus, Penicillium, Alternaria and Fusarium ${ }^{10,11}$. Of these fungal genera, Fusarium species were especially problematic ${ }^{12}$. Studies from otolaryngology reported that fungal colonization of the sinus cavity and upper airway is almost ubiquitous ${ }^{13-16}$. These studies found the same spectrum of fungal species with Aspergillus and Fusarium listed first and second in frequency. Many of these fungal species are highly resistant to anti-fungal agents especially Fusarium. Fusarium species produce a broad spectrum of mycotoxins with Fumonisins and Trichothecenes predominate ${ }^{12}$. Both these compounds have significant neurologic toxicity ${ }^{17}$. The problem of contamination of the human food chain by fungi and their mycotoxins has led to a world-wide industry to control the contamination of food (Romer Labs, Vicom Labs division Waters). Mycotoxin levels vary with environmental factors as well as storage techniques. There has been no successful way to prevent or remove these mycotoxins from food. This has left governmental authorities with few options. They have elected to monitor food supplies, set limits of exposure and discard food that exceed limits.

Animals fed hay or grain contaminated with mycotoxins develop serious problems that include neurologic deficits $^{18-20}$. Horses exposed to hay or grain contaminated with Fumonisins develop Equine Leukoencephalomalacia with paralysis of tongue, incoordination, ataxia, blindness, hind limb paralysis and eventual death with pathology finding severe brain damage.

The other dominant mycotoxins from Fusarium are Trichothecenes divided into four groups, A,B,C \& $\mathrm{D}^{12}$. The key to their toxicity is the presence of an epoxide ring moiety that generates free radicals setting off Oxidative Stress Reactions. They form covalent bonds to DNA, RNA and proteins. They target the 60S ribosome and peptidyl transferase shutting down protein production. The molecular weight of trichothecenes ranges from 200 to 500 Dalton. They are highly lipophilic able to be absorbed through skin, gut, crossing plasma membranes, including the blood brain barrier. Trichothecenes are known to accumulate in nervous tissue $^{17}$. In surveys of grain, the trichothecene Deoxynivalenol was the most common with T-2/HT-2 Toxins the fourth most common. Studies of Deoxynivalenol found that it could accumulate in tissues even when levels were below the regulatory limit ${ }^{21}$.

\section{Neurotoxicity \& Pathology of T-2 Toxins mimics ALS Pathology}

A review of T-2/HT-2 Toxins ${ }^{17}$ summarized the mounting evidence of its profound neurologic toxicity. The pathology of T-2 toxin mimics the pathology found in ALS. T-2 toxins are able to be absorbed across skin, mucosa and the blood-brain barrier accumulating in the CNS. They induce reactive oxygen species with oxidative stress reactions. They cause mitochondrial dysfunction and damage to a cascade of signaling pathways including p53, MAPK, Akt/mTOR, PKA/CREB and NF-kB with neuronal cell death. They cause perturbations of the mitochondrial respiratory chain with loss of ATP energy supplies. The motor neurons of the spinal cord have a relatively high demand for ATP.

\section{Immune Suppression due to Mycotoxins :}

Many mycotoxins cause damage to the immune system. The immunotoxicity of trichothecenes may be their most significant pathologic effect and T-2 toxin represents the most toxic of the trichothecenes. Li, M. et al in $2006^{22}$ found T-2 toxin impaired the mouse response to Reovirus suppressing immunoglobulin and interferon levels. Pestka, J.J. et al $2004^{23}$ found the trichothecene Deoxynivalenol and others caused a dose response suppression of immunologic markers. Studies by Obremski, K. et al $2013^{24}$ found T-2 toxin suppressed CD4, CD8 and CD21 levels in pig ileal Peyer's patches. Wu, Q. et al $2018^{25}$ reported downregulation of interferon, TGF-beta and Toll-like Receptors. There are similar immune deficits reported in ALS patients with subclass IgG deficiency ${ }^{26,27}$ and T-cell immune deficiency. Chronic low-dose poisoning of the immune system could evolve to create immune tolerance and immune evasion ${ }^{28,29}$. Immune evasion in ALS patients, could explain the recalcitrance to therapy. Monoclonal antibodies used in immunotherapy for oncology are being proposed 
as therapy for other diseases such as autoimmune disorders and chronic infections. If mycotoxins like T-2 toxin are causing immune evasion, then these new therapies could be promising in ALS.

\section{Neurologic Deficits Involving Both Upper and Lower Motor Neurons}

One of the clues to a diagnosis of ALS is the involvement of both upper and lower motor neurons. There is an insidious progression along the neuraxis. The pathology of trichothecenes could explain this pattern. Marcesca, M. $2013^{30}$ reports on the transport of food-associated trichothecenes from the gut to the brain. The ready movement of T-2 toxin across the blood brain barrier leads to its accumulation in the brain and spinal cord.

\section{Intranasal Pathways Bypass the Blood Brain Barrier and the CSF}

There is an intriguing possibility to explain the findings in ALS. If there is a focus of a fungal infection in the upper airway, especially the upper sinus cavity, the release of neurotoxic mycotoxins would rapidly move into both upper brain and lower spinal cord. There is a growing body of research documenting intranasal delivery of compounds and drugs into the brain and spinal cord. Thorne, R.G. et al $2004^{31}$ report on transport of IGF-1 to the brain from the nose of rats. Thorne, R.G. et al $2008^{32}$ report on the transfer of interferon-beta from the nose to the brain of monkeys. There is extensive research on intranasal absorption of insulin with the objective of replacing injection requirements. These studies found intranasal insulin was a viable route of delivery to the systemic blood stream. It also discovered that insulin was passing directly into the brain and spinal cord. Lochhead, J.J. et al $2019^{33}$ found insulin had neuroprotective effects given intranasally. It traveled along branches of the trigeminal nerve. Avgerinos, K.L. et al $2018^{34}$ found that intranasal insulin in 293 patients improved memory in patients with Alzheimer's or mild cognitive deficits. Rickels, M.R. et al $2016^{35}$ found that intranasal glucagon was effective in adults with type 1 diabetes mellitus in insulin induced hypoglycemia.

Studies by Pardridge et al $2011^{36}$ and Pardridge et al $2012^{37}$ document the passage of compounds by bulk flow along the course of the olfactory nerve to the olfactory bulb. Compounds enter into the brain at the level of the limbic system and forebrain. This would give rapid access to upper motor neurons. A second course from the sinus cavity follows two branches of the trigeminal nerve, ophthalmic and maxillary branches moving into the midbrain and spinal cord giving rapid access to lower motor neurons. Insulin $5.8 \mathrm{kDa}$ gains access to the CNS within 5-30 minutes.

\section{Pseudobulbar Affect :}

The rapid access to the limbic system and forebrain via the olfactory nerve pathway could help to explain pseudobulbar affect. Thakore, N.J. \& Pioro, E.P. $2017^{38}$ in Cleveland reported on pseudobulbar affect disorder found in 209 out of 735 ALS patients. They found an association of bulbar onset and dysfunction with predominant upper motor neuron disease. The ALS patients with pseudobulbar affect were younger in age with a shorter duration of disease. There was an association with the use of Baclofen, a surrogate for upper motor neuron dysfunction.

\section{Misconceptions of the Blood Brain Barrier and CSF :}

Pardridge et al $2012^{37}$ pointed out the misconceptions in the literature related to drugs and compound levels in the CSF. There was the misconception that CSF drug levels equated to levels in the brain and spinal cord. They found that CSF drug and compound levels were equivolent to systemic blood levels and not brain tissue levels. To assess brain and spinal cord drug levels required intracerebral microdialysis, functional MRI or PET scan.

\section{Biomarkers for ALS :}

The biomarkers listed for ALS are documented in many articles including Vu, L.T. \& Bowser, R. $2017^{39}$. Comparing these biomarkers for ALS with those found with mycotoxins finds similarities in multiple categories. Both lists include biomarkers for mitochondrial dysfunction and for immunologic deficits. An 
article by Vidal, A. et al $2018^{40}$ reviews the use of GC/mass spectroscopy to screen the world food supply for mycotoxins.

\section{Treatment:}

If ALS is due to mycotoxins generated by opportunistic fungi growing in patients, then treatment would require aggressive control of the fungal infection. Fusarium species are prime suspects presenting the added problem of resistance to almost all the standard antifungal agents. Voraconazole has shown some promise controlling Fusarium species. The literature out of Brazil, Herkert, P.F. et al $2019^{41}$ suggest combined therapy with Amphotericin B and Voraconazole. A large part of the resistance of these fungi could be due to the profound immune suppression caused by the mycotoxins. Treatment with plasma exchange would help to lower the body burden of toxins. More selective treatment with affinity columns or activated charcoal dialysis could help. The effort to overcome the immune resistance could require gammaglobulin, interferon and/or interleukin therapy. One of the most intriguing possibilities is the use of immunotherapy. The new monoclonal antibodies might reverse immune suppression such as PD-1/PD-L1 inhibitors ${ }^{28,42}$. Finally, there needs to be a search for the focus on infection with an effort to remove it.

William K. Reid Date

1. Wolfgram F, Myers L. Amyotrophic lateral sclerosis: effect of serum on anterior horn cells in tissue culture. Science.1973;179(4073):579-580.

2. Field EJ, Hughes D. Toxicity of motor neurone disease serum for myelin in tissue culture. $\mathrm{Br} \mathrm{Med} J$. 1965;2(5475):1399-1401.

3. Silani V, Scarlato G, Valli G, Marconi M. Plasma Exchange Ineffective in Amyotrophic Lateral Sclerosis. Archives of Neurology.1980;37(8):511-513.

4. Reid W. Immunosuppression \& Mycotoxins Causing Amyotrophic Lateral Sclerosis. the Winnower. Published 2017. Accessed.

5. Bennett JW, Klich M. Mycotoxins. Clin Microbiol Rev.2003;16(3):497-516.

6. Eskola M, Kos G, Elliott CT, Hajslova J, Mayar S, Krska R. Worldwide contamination of food-crops with mycotoxins: Validity of the widely cited 'FAO estimate' of 25. Crit Rev Food Sci Nutr. 2019:1-17.

7. Gruber-Dorninger C, Jenkins T, Schatzmayr G. Global Mycotoxin Occurrence in Feed: A Ten-Year Survey. Toxins (Basel).2019;11(7).

8. Auchtung TA, Fofanova TY, Stewart CJ, et al. Investigating Colonization of the Healthy Adult Gastrointestinal Tract by Fungi.mSphere. 2018;3(2).

9. de Hoog S, Monod M, Dawson T, Boekhout T, Mayser P, Gräser Y. Skin Fungi from Colonization to Infection. Microbiol Spectr.2017;5(4).

10. Al-Jaal BA, Jaganjac M, Barcaru A, Horvatovich P, Latiff A. Aflatoxin, fumonisin, ochratoxin, zearalenone and deoxynivalenol biomarkers in human biological fluids: A systematic literature review, 2001-2018. Food Chem Toxicol. 2019;129:211-228.

11. Omotayo OP, Omotayo AO, Mwanza M, Babalola OO. Prevalence of Mycotoxins and Their Consequences on Human Health. Toxicol Res.2019;35(1):1-7.

12. Dejardins AE. Fusarium Mycotoxins: Chemistry, Genetics and Biology. The American Phytopathological Society, St. Paul, Minnesota; 2006.

13. Braun H, Buzina W, Freudenschuss K, Beham A, Stammberger H. 'Eosinophilic fungal rhinosinusitis': a common disorder in Europe? Laryngoscope. 2003;113(2):264-269. 
14. deShazo RD, Chapin K, Swain RE. Fungal sinusitis. N Engl J Med. 1997;337(4):254-259.

15. Ponikau JU, Sherris DA, Kern EB, et al. The diagnosis and incidence of allergic fungal sinusitis. Mayo Clin Proc. 1999;74(9):877-884.

16. Waitzman AA, Birt BD. Fungal sinusitis. J Otolaryngol.1994;23(4):244-249.

17. Dai C, Xiao X, Sun F, et al. T-2 toxin neurotoxicity: role of oxidative stress and mitochondrial dysfunction. Arch Toxicol.2019;93(11):3041-3056.

18. Gabal MA, Awad YL, Morcos MB, Barakat AM, Malik G. Fusariotoxicoses of farm animals and mycotoxic leucoencephalomalacia of the equine associated with the finding of trichothecenes in feedstuffs. Vet Hum Toxicol. 1986;28(3):207-212.

19. Raymond SL, Smith TK, Swamy HV. Effects of feeding a blend of grains naturally contaminated with Fusarium mycotoxins on feed intake, metabolism, and indices of athletic performance of exercised horses. $J$ Anim Sci. 2005;83(6):1267-1273.

20. Riet-Correa F, Rivero R, Odriozola E, Adrien Mde L, Medeiros RM, Schild AL. Mycotoxicoses of ruminants and horses. $J$ Vet Diagn Invest. 2013;25(6):692-708.

21. Mishra S, Srivastava S, Dewangan J, Divakar A, Kumar Rath S. Global occurrence of deoxynivalenol in food commodities and exposure risk assessment in humans in the last decade: a survey. Crit Rev Food Sci Nutr. 2019:1-29.

22. Li M, Cuff CF, Pestka JJ. T-2 toxin impairment of enteric reovirus clearance in the mouse associated with suppressed immunoglobulin and IFN-gamma responses. Toxicol Appl Pharmacol. 2006;214(3):318-325.

23. Pestka JJ, Zhou HR, Moon Y, Chung YJ. Cellular and molecular mechanisms for immune modulation by deoxynivalenol and other trichothecenes: unraveling a paradox. Toxicol Lett.2004;153(1):61-73.

24. Obremski K, Podlasz P, Zmigrodzka M, et al. The effect of T-2 toxin on percentages of CD4+, CD8+, CD4+ CD8+ and CD21+ lymphocytes, and mRNA expression levels of selected cytokines in porcine ileal Peyer's patches. Pol J Vet Sci. 2013;16(2):341-349.

25. Wu Q, Wu W, Franca TCC, Jacevic V, Wang X, Kuca K. Immune Evasion, a Potential Mechanism of Trichothecenes: New Insights into Negative Immune Regulations. Int J Mol Sci. 2018;19(11).

26. Ostermeyer-Shoaib B, Patten BM. IgG subclass deficiency in amyotrophic lateral sclerosis. Acta Neurol Scand.1993;87(3):192-194.

27. Beers DR, Zhao W, Wang J, et al. ALS patients' regulatory T lymphocytes are dysfunctional, and correlate with disease progression rate and severity. JCI Insight. 2017;2(5):e89530.

28. Qin W, Hu L, Zhang X, et al. The Diverse Function of PD-1/PD-L Pathway Beyond Cancer. Front Immunol. 2019;10:2298.

29. Schönrich G, Raftery MJ. The PD-1/PD-L1 Axis and Virus Infections: A Delicate Balance. Front Cell Infect Microbiol. 2019;9:207.

30. Maresca M. From the gut to the brain: journey and pathophysiological effects of the food-associated trichothecene mycotoxin deoxynivalenol. Toxins (Basel). 2013;5(4):784-820.

31. Thorne RG, Pronk GJ, Padmanabhan V, Frey WH, 2nd. Delivery of insulin-like growth factor-I to the rat brain and spinal cord along olfactory and trigeminal pathways following intranasal administration. Neuroscience. 2004;127(2):481-496.

32. Thorne RG, Hanson LR, Ross TM, Tung D, Frey WH, 2nd. Delivery of interferon-beta to the monkey nervous system following intranasal administration. Neuroscience. 2008;152(3):785-797. 
33. Lochhead JJ, Kellohen KL, Ronaldson PT, Davis TP. Distribution of insulin in trigeminal nerve and brain after intranasal administration.Sci Rep. 2019;9(1):2621.

34. Avgerinos KI, Kalaitzidis G, Malli A, Kalaitzoglou D, Myserlis PG, Lioutas VA. Intranasal insulin in Alzheimer's dementia or mild cognitive impairment: a systematic review. J Neurol. 2018;265(7):1497-1510.

35. Rickels MR, Ruedy KJ, Foster NC, et al. Intranasal Glucagon for Treatment of Insulin-Induced Hypoglycemia in Adults With Type 1 Diabetes: A Randomized Crossover Noninferiority Study. Diabetes Care. 2016;39(2):264-270.

36. Pardridge WM. Drug transport in brain via the cerebrospinal fluid.Fluids Barriers CNS. 2011;8(1):7.

37. Pardridge WM. Drug transport across the blood-brain barrier. $J$ Cereb Blood Flow Metab. 2012;32(11):1959-1972.

38. Thakore NJ, Pioro EP. Laughter, crying and sadness in ALS. J Neurol Neurosurg Psychiatry. 2017;88(10):825-831.

39. Vu LT, Bowser R. Fluid-Based Biomarkers for Amyotrophic Lateral Sclerosis. Neurotherapeutics. 2017;14(1):119-134.

40. Vidal A, Mengelers M, Yang S, De Saeger S, De Boevre M. Mycotoxin Biomarkers of Exposure: A Comprehensive Review. Compr Rev Food Sci Food Saf. 2018;17(5):1127-1155.

41. Herkert PF, Al-Hatmi AMS, de Oliveira Salvador GL, et al. Molecular Characterization and Antifungal Susceptibility of Clinical Fusarium Species From Brazil. Front Microbiol. 2019;10:737.

42. Boussiotis VA. Molecular and Biochemical Aspects of the PD-1 Checkpoint Pathway. N Engl J Med. 2016;375(18):1767-1778. 